\title{
The Aesthetic Actualization of Gestalt Image in English Translation of Chinese Poems
}

\author{
Chong $\mathrm{Su}^{1 \& 2}$ \\ ${ }^{1}$ College of International Study, Southwest University, Chongqing, China \\ ${ }^{2}$ School of Foreign Languages, Southwest Petroleum University, Chengdu, China \\ Correspondence: Chong Su, School of Foreign Languages, Southwest Petroleum University, Chengdu, China. \\ E-mail: 1336380317@qq.com
}

\author{
Received: November 24, 2017 Accepted: December 12, 2017 Online Published: December 23, 2017 \\ doi:10.5539/ijel.v8n2p233 URL: http://doi.org/10.5539/ijel.v8n2p233
}

Program information: This paper is the research achievement of a key program supported by Educational Commission of Sichuan Province of China; The key program name: 认知心理学视域下意象翻译的语义场研究 (Research on semantic field of image translation from the perspective of cognitive psychology).

\begin{abstract}
As significant schools in contemporary cognitive science, gestalt psychology and cognitive linguistics provide the conceptual model and theoretical framework for the multidimensional research on cognitive translation study. Their major theoretical perspective makes them as the methodological principles of gestalt image study in cognitive translation which could be viewed from both mental and linguistic perspectives. The paper focuses on making a tentative and exploratory study of the aesthetic actualization of gestalt image in English translation of Chinese poems and thus aims to discover the conceptual mental experience of actualizing aesthetic quality of image-G through cognitive processing in English translation of Chinese poems.
\end{abstract}

Keywords: gestalt psychology, image-G, Chinese poems translation, aesthetic actualization

\section{Introduction: Image-G in Chinese Poems}

In the studies of Chinese poems, the notion "image" is generally considered as a connotation which is culturally-embedded in various essential elements in literary poems, such as words, phrasal idiom, quotation, idiomatic expression, and figurative expression. Image is an iconic imagery of the composition of perceptive and literary appreciation, a dynamic participation of the writer's inner beings and intuition in the process of artistic creation (Wang, 2013). A Chinese poem is generally composed of a complicated concatenation of images that are embedded within text to create profuse comprehension and understanding that can surpass what is derived from text alone.

According to Gestalt psychology, Image-gestalt (image-G) is a psychological literary term, referring to a unified image regarded as a unit whose connotation cannot be simply concluded from the sum of each parts and whose whole implication is more than the merely sum of its parts (Jiang, 2002). Image-G is a ubiquitous possession in Chinese poems which constructs cognitive-psychological aesthetic image rather than solitary items. As an artistic object, Chinese poems possess image in the gestalt sense of holistic quality, which is the combination of linguistic expressions and mental representation. When referring to the transmitting of aesthetic essence of Chinese poems, the recreation of image-G from Chinese to English is a major concern facing literacy translators and translation researchers. The interpretation of image-G undoubtedly will affect readers' psychological and sensory organoleptic experience in their aesthetic appreciation of Chinese poems. Literary translation is not only to transfer to Chinese language into English, but also to evoke readers' same aesthetic experience by conveying the psychological culture factors in English. As far as the psycho-physical patterns are concerned, the aesthetic value is supposed to be transferred much more precisely if translation strategy could be informed by theories of gestalt psychology (Xu, 2006). 


\section{Aesthetic Perception of Image-G in English Translation of Chinese Poems}

\subsection{Aesthetic Perception of Image-G as an Inbred Faculty}

Gestalt psychology has emphasized the significance of human's organization of cognitive perception in principle: our aesthetic perception experiences are unified wholes rather than bunches of constituting elements, which is perceived as a natural organization of a gestalt (Koffka, 2015). A translator will not discern images in literary texts so effortfully when he captures image-G visual pattern, In other words, a translator's embedded cognitive capability for paraphrasing and appreciating literary aesthetic essence in Chinese poems can be identified with language-producing proficiency, constructional psychological concentration, psychological faculty of perception and constituted aesthetic actualization and so on. In addition, divergent senses of aesthetic functions are originated from a great variety of delicate image-G. Images-G perception is to some degree an innately aesthetic mechanism, which is a comprehensive projecting module of one's operation of knowledge, cognitive and aesthetic schema (Jiang, 2008).

A reader of English translation of Chinese poems has experience of certain aesthetic qualities as well. To perceive the gestalt image is to sketch the psychological contours of poetic subjects and to experience the same aesthetic gestalt of an author. A reader will not perceive images by interpreting translation vision so effortfully when he seizes image-G mental pattern. When at the same time the translator accumulates from textual information which he synthesizes into a whole, he comes to formulate an artistic image, which is called as an aesthetic model of perception (Bell, 1991). In other words, people's gestalt perception as an inbred faculty helps them to apprehend the holistic structured image-G instead of individual and incoherent gestalt items in comprehending English translation of Chinese poems.

\subsection{Aesthetic Perception of Image-G as an Instruction for Cognitive Linguistic Actualization}

A text of English translation of Chinese poems can be comprehensibly apprehended when referential intertextuality is brought into effect in working cognitive linguistic construction. Aesthetic perception of image-G in cognitive linguistic construction is the psychological functioning which is nothing less than mental actualization and cognitive analysis of a text's logic structure. The instruction prompts readers to perceive the entirety without their recognition of the systematic parts, that is, it causes readers to respond to certain gestalt image subject to gestalt holism in the text of English translation of Chinese poems (Barnstone, 1993). What makes systematic parts is not the discrete configuration itself, rather the aesthetic connotation of image-G relating to a certain context of English translation of Chinese poems despite its aesthetic realization is profoundly embedded.

According to Charles J. Fillmore, scene-and-frames notion is the amalgamation of linguistic grouping and particular meaningful setting which is associated with linguistic unity (Fillmore, 1996). The notion also accounts for the aesthetic perception of image-G in literary appreciation since its coherent internal artistic scheme is concerned. That is, all the particular environmental setting realized by the linguistic form is the image-G of a meaningful setting induced before a reader's cognitive perception. All the image- $G$ in the literary text is corporeally brought about linguistic form, namely, the frame. Thus image- $G$ is first of all actualized by an integrated and undeviating linguistic frame and then comes out a scene image affected by perceptional situation. As an instruction, aesthetic perception of image-G permeates readers' cognitive processes and psychological-processing when readers endeavor to create a coherent mental presentation of image- $G$ in comprehending English translation of Chinese poems. Chinese poems contrasts with other foreign literature works that its linguistic schema is with indistinct essence. Every piece of Chinese poems as image-G amalgamated with atmosphere and tone are hazy, modifying and ill-defined, and thus how translators and readers actualize it does produce effects. Linguistic structure in the Target-text may come out as equivalent with that of the ST (source text), which, however, does not only rise from correspondence-finding of linguistic items. Thus, it is the consequence of cognitive predisposition and linguistic all-roundness.

\section{Cognitive Diagram Dimension of Image-G in English Translation of Chinese Poems}

Image- $G$ is the core basic-divergent constituent in the complement structure of poetic value based on the proposition that linguistic iconicity is the cognitive representational referent of image in discourse. The modality of discourse maps conceptual construction by the way of diagram. However, linguistic iconicity cannot be regarded as equally good example of linguistic structure and conceptual construction are simply corresponding, yet the configuration of linguistic iconicity is quite complicated by its nature. The image- $G$ in Chinese poems is the product of conceptualization, which can be considered as a sensation of perception or cognitive experience beyond sensory perception. The application of cognitive linguistic theories to the appreciation of image-G in textual space is essential for the understanding of diagram dimension in cognitive poetics and stylistics. 


\subsection{Profiling of Image-G in Textual Space}

As for image-G in textual expression, its semantic information is the construal imposed on a specific scenario. One key manifestness of construal is that "base" is lexicalized to represent "profile", which can be defined as conceptual networks structured in terms of conceptual domains required by image-specific semantics. Langacker claimed that, "profile" is the highlighting depicted partial in "base" to some extent. In other words, "profile" is an entity designated by image categories and a focal spot in conceptualization schema (Langacker, 1987). Obviously, "base" is of the utmost requisite in the semantic value of image-G categories, though "base" itself cannot constitute any semantic value. A consequence of the claim is that "base" is a latent network composed of cognitive domains which is activated by the interpretation of image- $G$ in textual space. Thus the assertion will attach practicable research guidance and far-reaching theoretical significance to the study of aesthetic actualization of gestalt image. Take an example from the translation of Meng Jiao's poem A Traveller's Song (孟 郊《游子吟》):

慈母手中线, 游子身上衣。

临行密密缝, 意恐迟迟归。

谁言寸草心, 报得三春晖。

cimu shouzhong xian, youzi shenshang yi.

linxing mimi feng, yikong cici gui.

shuiyan cuncao xin, baode sanchun hui.

“A thread is in my fond mother's hand moving.

For her son to wear the clothes are leaving.

With her whole heart she's sewing and sewing.

For fear I'll ever be roving and roving.

Such life-long mother's love how may

One simple little heart repay?"

(Translated by W. J. Fletche)

The profiling of aesthetic ideology as holistic image- $G$ in the Chinese version is presented by two ideation bases: filial piety and maternal love. One active ideational metaphor relates to the contents of filial piety that results in inch-long grass while the other corresponds to the gentle sunshine. The two ideational metaphors show that how active ideational metaphors are on operation in textual space and enable base and semantics frame to become associated with each other and constructed at the conceptual level. "plant" and "natural attractions" frame in S-text are of a profection mapping, where filial piety and maternal love are conceptualized in terms of sentiment. Inch-long grass and gentle sunshine contain the semantics value of "infinitesimal" and "warmth and kindness" respectively, which are being highlighted as the focal spots to map the image-G: how profound maternal love is and how humble filial piety will be. In the English vision, the target frame of the two ideational metaphors is rendered into sentiment as "mother's love" and "heart repay" directly, without paraphrasing the source frame, "plant" and "natural attractions" of ST. It can thus be safely assumed that the "profile" of image-G in ST and TT (target text) is equally matching, whereas the "base/frame" which present cognitive context for its "profile" is not alike at all.

\subsection{Specificity of Image-G in Textual Space}

As the second cognitive diagram dimension, specificity posits a decisive influence over the level of accuracy rating and final diagram of image-G. Like the other dimensions, it is entirely relative in nature with respect to the semantic primes in textual space. Although proper degree of specificity is determined by relevant context in specific conceptualization process, it hinges ultimately on the conceptualization figure, which is subject to certain textual intercourse and intention. In general, insufficient detailed and definite textual description will reemphasize omitted characteristics of image-G, whereas excessive detailed and definite textual description will help manifest implied and new characteristics (Tabakowska, 1993). As a result, the higher specificity level it is attached to textual description, the more cognitive domains of relatively inferior specificity level will be activated. That is to say, any exiguity choice of specificity level applied to literary text has decisive influence on the cognitive construction of image-G. Take an example from the translation of Li Bai's poem Waiting in Vein (李白《怨情》): 
美人卷珠帘, 深坐歳蛾眉。

但见泪痕湿, 不知心恨谁。

meiren juan zhulian, shenzuo cu e'mei.

danjian leihen shi, buzhi xinhen shui.

"Beautiful is this woman who rolls up the pearl-reed blind,

She sits in an inner chamber,

And her eye brows, delicate as a moth's antennae.

Are drawn with grief.

One sees only the wet lines of tears

For whom does she suffers this misery?

We do not know."

(Translated by Amy Lowell)

The first two lines depict an image of an aggrieved beauty with contracted brown without relaxation who is in her inner chamber decorating with delicately beads bed hanging. From the perspective of specificity dimension, $z h u$ "pearl", shen "inner" and $e$ "moth" can be regarded as the textual description of relatively superior specificity level of the image-G connected with drop curtain, sitting and brown, whose implication and undertone is underlying and cognitive schematized. By conceptualizing the content dimension of the superior elements, which functions within a mapping scope based on image schema and linked by a shared conceptual experience of image-G, Amy translated the relatively superior elements into "pearl-reed blind", "in an inner chamber" and "delicate as a moth's antennae" to make them become "instantiation" and come closer to represent specificity of image-G in the original. The translation substantiates the comparatively abstract implication of $z h u$ "pearl", shen "inner" and $e$ "moth", makes them schematized, and renders vogue images in the original more fine-grained and elaborated.

\subsection{Prominence of Image- $G$ in Textual Space}

Prominence is the comparatively highlighting level of various conceptual parts in a coherent image-G whole and relational prominence shares the closest correspondence with the image- $G$ in English translation of Chinese poems. The psychological organization role for relational prominence turns out to be asymmetric, which is a typical figure/ground organization. Like reversible visual scenes such as the face/vase illusion, the cognitive aptitude to distinguish figure from ground in image- $G$ whole in textual space is also reflected in the scenario construction which is restricted by cognitive processing in original text translation. Any semantic and aesthetic component who can constitute a cognitive reference point will be listed as preferred "ground" and the others who deviate from holistic organization will be put on the position as "figure" of image-G (Kuang, \& Wen, 2003). The conception of figure and ground is applicable to the construal of varied tense, style, theme and aesthetic actualization item of image-G between ST and TT in English translation of Chinese poems. The discrepancy in the prominent figure and figure/ground organization tends to present a covert aspect and is associated with pragmatic practice in literary translation. Take an example from the translation of Cao Zhi's poem A Groan (曹 植《七哀》):

明月照高楼, 流光正徘徊。上有愁思妇, 悲叹有余哀。

借问叹者谁, 言是宕子妻。君行逾十年, 孤妾常独栖。

君若清路尘, 妾若浊水泥。浮沉各异势, 回合何时谐?

愿为西南风, 长逝入君怀。君怀良不开, 贱妾当何依。

mingyue zhao gaolou, liuguang zheng paihuai. shangyou chou sifu, beitan you yu'ai

jiewen tanzhe shui, yanshi dangzi qi. junxing yu shinian, guqie chang duqi.

junruo qing luchen, qieruo zhuo shuini. fuchen ge yishi, huihe heshi xie?

yuanwei xinan feng, changshi rujun huai. junhuai liang bukai, jianqie dang heyi.

"Softly on the tower streams of light play;

It seems the moon is loath to move away.

For here is beauty wilting, tender sighs 
Telling of a tender heart in pain, which cries.

May we ask who is there so full of ruth?

A wife in name, a window, ah, in truth!

You are far, far away for o'er ten years;

I an alone, alone and oft in tears.

You're like the dust drawn upward on the way;

Like mud in dirty water still I stay.

One sinking, the other swimming we remain.

If ever, when are we to meet again?

Would that I were the wind from the southwest,

That I could rush across to your breast!

From your embrace, if you should shut me out,

Where should I go? Where should I roam about?"

(Translated by Xu Yuanchong)

The fitst two lines in the S-text portray an image of tranquil moonlight who sleeps upon and casts a gentle light over the tower. In terms of scenario description of image-G, the translation vision is almost equivalent to ST on account of the correspondence with ST about aesthetic truth value. However, in the frame of prominence dimension, mingyue "bright moon" is the figure, liuguang "streams of moonlight" is the ground and gaolou "tower" is just a part of underlying background in the figure/ground organization of image-G schema in ST. What sets it apart from ST is that gaolou "tower" is highlighted to the position as figure which occupies the focal spot and mingyue "bright moon" is melt invisibly into the background in TT. Thus a completely homogeneous image-G schema in literary translation is not just the copy of a mental picture of prominence organization diagram in ST, nor solely the reflective structuring of translators' conceptual perception about the original's prominence functioning, but rather from recurring interaction of a conceptual-affective reproduction between ST and translators' prominence organism.

\subsection{Perspective of Image-G in Textual Space}

The dimension of perspective refers to conceptualizing corpuses' angle of vision from which textual space is examined. The specific frame of reference corpuses adopt in the perception of image-G of English translation of Chinese poems is also based on cognitive process of conceptual structuring. In general, perspective ranges from viewpoint, deixis, subjectivity to objectivity, which reflects embodied concepts of encoded and externalized scenes in textual space (Langacker, 1987). Viewpoint exerts influence on the typical cultural presuppositions and conventional presuppositi patterns of conceptualizing corpuses, which is determined by semantic distinctions and cultural relevance. Subjectivity is the "degree to which an entity functions asymmetrically as the subject or the object of conception" (Langacker, 1998, p. 592), which conceptualizes discourse event scenario of image-G in the frame of textual space. Deixis is "the phenomenon of using elements of the subject's situatedness - more specifically, the subject qua speaker in speech event — to designate something in the scene" (Croft \& Cruse 2004: 59), which bears the core element in image-G construal functioning of its conceptual organization dimension of perspective. Take an example from the translation of Li Dan's writing Lao-tse (李姍《老子》):

知不知, 上。不知知, 病。

zhubuzhi, shang. buzhizhi, bing.

知不知, 上。不知, 知, 病。

zhubuzhi, shang. buzhi, zhi, bing.

知, 不知, 上。不知, 知, 病。

(以) 知 (为) 不知, 上,

(wei)zhi(wei)buzhi,shang.

(以) 不知 (为) 知, 病。

(wei)buzhi(wei)zhi,bing.

"To know the unknowable, that is elevating. 
Not to know the knowable, that is sickness."

(Translated by Carus. W. C)

"To know when one does not know is best.

To think one knows when one does not know is a desire desease." (Translated by Waley. A)

"To know, but to be as through not knowing, is the height of wisdom.

Not to know, and yet to affect knowledge, is a vice."

(Translated by Giles. L)

"To regard knowledge as no-knowledge is best.

To regard no-knowledge is sickness."

(Translated by Wu Jingxiong)

The comparison between several different translation visions of Lao-tse selected text embodies the difference not only in syntactic analysis of TT, but also in the voluntary intervention on perspective dimensions of image-G by the four translators as conceptualizing corpuses in interpretation framework. The translators' conceptual prospective construal of image-G roots from physical force interactions within sentiment orientation and inter-psychological manner: The four different translations of buzhi "unknowable / notknowing / no-knowledge / not to know" has presented evidence for varied cognitive instruments which are used by four translators for non-linguistic mental processing and are emerged from their experiences of interacting with the world; From the standpoint of wholly corporeally positioning, the four translators are in a certain approaching status in the conceptualization notion of zhi, buzhi, (wei)zhi, (wei)buzhi, bing and shang, which carries semantically image-G of its own and is related to the corresponding insight into cognitive networks in a established perpendicular direction of basic human scheme; From a intercourse frame of reference, the dimension of deixis is defined by the four translators' cognitive environment and secular space and their cognitive models in the discourse frame interpretation. Moreover, their approaches to the semantic domains of inference as empathy and their characteristically manifested building of the ST is also in an appropriate sense of subjectivity.

\subsection{Metaphor of Image-G in Textual Space}

A kind of conventional viewpoint is that metaphor is a figure of speech and an imaginative way in which a word or phrase that ordinary designates one thing is uesd to designate another, thus making an implicit comparison. As a matter of fact, metaphor is also a key realization and dimension in the construal operation of image-G. Thus, representing image- $\mathrm{G}$ of a specific cognitive domain setting by the dimension of metaphor can be regarded as construing the functional semantic structure of the cognitive domain by the metapher's cognitive and experiential model. As Snell-Hornby points out, "As an abstract concept, metaphor might be universal (as claimed in); in its concrete realization however, being closely linked with sensuous perception and culture-bound value judgement, it is undoubtedly complicated by language-specific idiosyncrasies" (Snell-Hornby, 1995: 62). From the perspective of cognitive metaphor dimension, the translatability of image-G in English translation of Chinese literature is decided by the degree of coincidence between the cognitive experience and cognitive models of the source language and the target language. All in all, different languages may contain varying level of metaphorical exuberance and metaphorical cultural-overlapping blending in literary translation under controlled event-frame conditions, which could be easily and unequivocally represented in the format of a sequential scenario by the construction of the dimension of metaphor. Take an example from the translation of Cao Xueqin's great works of literature A Dream in Red Mansions (曹雪芹《红楼梦》):

黛玉听了这话, 如轰雷掣电, 细细思之, 竟比自己肺腑中掏出来的还觉恳切。

Daiyu tinle zhehua, ru honglei chedian, xixi sizhi, jingbi ziji feifu zhong taochulai de haijue kenqie.

"These words made Lin Tai-yue feel as if she had been blasted by thunder, or struck bt lightning. But after carefully weighing them within herself, they seems to her far more fervent than that might have emanated from the depths of her own heart."

\section{(Translated by Yang Xianyi)}

The conceptual metaphor of feifu zhong taochu in the original can arouse translator's immense imagination and play a vital role in creating "G-image beyond images" of the whole ideorealm. The source concept (the tenor) is internal organs of human body, the target concept (the vehicle) is the abstract concept of emotion and the mapping scope is that emotion can be stored in the internal organs of human body. This means that the instantiation of conceptual metaphor is defined by language-specific and culture-specific models. There are 
enormous phrases and idioms containing the images of internal organs, which are linked by a shared conceptual structure and are reflected from a physiological angle in the degree of conventionalization a metaphor has achieved in a speech community. Yet internal organs are much less semantically associated with emotion, sensation and perception in English expressions. Yang Xianyi's interpretation is almost the cognitively aesthetic equivalent of the language-specific and culture-specific models in the originals by activating the conventional units of internal organs. The general case of this seems to be that Chinese language contains much less volume of image-G in metaphorical as well as metaphorical exuberance than English language does in literary works, particularly in the conceptualization notion of strong emotion expression. The English translation of metaphors in Chinese ST tends to be excessively deficient and insufficient, thus it is necessary for translators to modify image schemata and their metaphorical projections in an appropriate sense of objectivity in English translated text.

\section{Aesthetic Actualization of Image-G in English Translation of Chinese Poems}

\subsection{Aesthetic Essence in English Translation of Chinese Poems}

Chinese poems is fundamentally characterized by varied themes, variable modes, elegant verbalization and exquisite aesthetic image. Thus, a refined version of Chinese poems is not only the linguistic and rhetorical representation, but also the cognitive and aesthetic actualization of original text due to the fact that every element of Chinese poems is a scenes-and-frames integration. Aesthetic essence in text is evoked by the way how translators mentally visualize it in the ST as well as reconstruct and actualize it in the TT being produced. As aesthetic metaphrase, English translation of Chinese poems is that translators artistically comprehend the image structure in ST and then appropriately reconstruct the aesthetic image and conceptual system so that target readers may be inspired, moved and aesthetically entertained in the same way as one reads the original. Take an example from the translation of Wang Wei's poem One Mission to the Frontier (王维《使至塞上》):

单车欲问边, 属国过居延。孤篷出汉塞, 归雁入胡天。

大漠孤烟直, 长河落日圆。萧关逢候骑, 都护在燕然。

danche yu wenbian, shugo guo juyan. gupeng chu hansai, guiyan ru hutian.

damo guiyan zhi, changhe luori yuan. xiaoguan feng houqi, duhu zai yanran.

"A single carriage sets off for the bonder,

Journeying past the subject state of Juyan;

On we jolt, leaving Han fortress behind,

A wild goose winging back to the Hunnish sky.

In the great desert one straight plume of smoke,

By the long river at sunset a ball of flame.

Before Xiao Pass we meet a mounted patrol

And learn that our forces have taken Mount Yanran"

(Translated by Yang Xianyi)

The fifth and sixth lines portray a true wonder of nature of spectacular desert scenery near the frontier fortress, which arouses visual beauty and pleasant sensation by vivid image spacing description: The size type is characterized by the fierce contrast of damo "great desert" and guiyan "smoke" as well as changhe "long river" and luori "sunset". This view that a holistic system specific to Chinese aesthetic essence has evolved contrasted with $z h i$ "straight" and yuan "a ball", which makes picturesque features of guiyan "one straight plume of smoke" and luori "sunset a ball of flame". Yang Xianyi mentally visualized the aesthetic essence of the original at first and then built up a stereoscopic picture by putting guiyan "smoke" into one straight plume of smoke, luori "sunset" into sunset a ball of flame: "straight plume" is an exact recapture of the smoke rising into the air in a shape of a feather by a complete contrast to "great desert"; "a ball of flame" accurately portrays the fashion and hue of luori "sunset" by a complete contrast to "the long river", which can effortlessly arouse readers' asymmetrical striking aesthetic experiences of a high degree accordance with the ST.

\subsection{Aesthetic Equivalence and Image- $G$}

The actualization of image-G in English translation of Chinese poems brings into light underlying aesthetic qualities and thus reaches to holistic correspondence in its equivalent. It is embodied in the following procedure modes: the aesthetic equivalence of image-G reproduction is realized in the continuous interaction between 
translators and the S-text through flexible application of right aesthetic operation such as imaginative and figurative faculties. With full resources of the aesthetic representation and the unified concept of image-G transformation schema ready in memory, translators realize the formulation of image-G functions (Gibbs, R. 1995). Then translators make use of the image- $G$ to assemble various aesthetic values and ideologies to synthesize into an integral whole in TT representation, aesthetic value and standard and ideology and so on. Any gestalt image in the imagery structure can be properly actualized in the ST to preserve the original imagery structure and to acquire matching aesthetic equivalence. Thus readers can interpret the $\mathrm{ST}$ in a similar linguistic frame and have an equivalent aesthetic experience. Take an example from the translation of Si Kongtu's writings Twenty-four Poetry Style (司空图《二十四诗品》):

绿衫野屋, 落日气清, 脱巾独步, 时闻鸟声。鸿雁不来, 之子远行。

所思不远, 若为平生。海风碧云, 夜渚月明, 如有佳语, 大河前横。

lvshan yewu, luori qiqing, tuojin dubu, shiwen niaosheng.

hongyan bulai, zhiziyuanxing. suosi buyuan, ruowei pingsheng.

haifeng biyun, yezhu yueming, ruyou jiayu, dahe qianheng.

"A hut in the wood green.

Sun setting, an aura serene.

In casual dress for a walk alone.

Bird's song steeped in twilight's tone.

The bosom friend in strange land will stay.

No tidings by wild geese, to one's dismay.

Yet the image always seems not far away,

And as intimate as in the good old day.

Clouds set in commotion by a sea gale.

The creek lies still in moonlight pale.

Suddenly a river looms large across,

The moment some poetic insight crops."

(Translated by Xie Yi)

The writings bring out the image-G as a whole by three textual functions in ST. Indeed, the three textual functions seem like a very cooperative organization, in which the partners are fully "link-and-comes" to each other. The first four lines present an invigorating autumn climate by describing a variety of natural scene images, which implies the systemic-functional image-G as the author's intoxicating joyfulness with harmonious coexistence with nature. The fifth and sixth lines demonstrate that the author is in a despondent mood because he is not keeping on shut cocking letters with a time friend, which implies the systemic-functional image-G as drizzling melancholy and thickening yearning in the author. The last four lines take on the picture that clouds are pale and a light breeze is blowing by drawing an open and broad landscape, which implies the systemic-functional image- $G$ as the author's smoothing equanimity by placing the longing for the friend into the fine scenery.

Xie Yi formulates a mental conception of the aesthetic veining of image-G out of the ST at first and then interprets the three successive diagram framework to give rise to a high degree of aesthetic equivalence: "sun setting", "an aura serene" and "stepped in twilight's tone" manifest itself in the scene of lonesome mountains when birds are singing and expresses the real coherence emotion of cheerfulness as ST. "To one's dismay" has precise aesthetic equivalent to emotionally distracted and listless mind in ST. "As intimate as in the good day" and "the moment some poetic insight crops" can be made accessible to readers into epiphanic insight with the transcendental spiritual solace from the communion with natural beauty in the original image-G.

\subsection{Aesthetic Quality of Harmony and Image-G}

As an omnipresent theoretical conception, harmony characterizes many forms of art as an aesthetic quality. Through its specific image schema, the artistic arrangement of aesthetic essentials in Chinese poems is harmonized and comes to be aesthetic actualities which utter and regulate its organization by the principle of harmony. As Paul M. Dufrenne writes about this conception: “... the sensuous must be arranged and ordered in 
such a way as to be perceived unequivocally...", it determines whether the function of the source text or passages in the source text can be preserved or have to be adjusted or even altered (Dufrenne, 2002). Quality of harmony is represented through the entire translation process in translators' aesthetic actualization of image-G. It is through the harmony that the author creates the literary works of art and that translators manage to grasp the gestalt image and reproduce the original. Therefore, the harmony is an aesthetic impact that translators attempt to achieve, and an aesthetic principle that translators endeavor to comply with. Every piece of Chinese literary works is image-G, which is rebuilt mentally by the translator and then actualized linguistically in the target. Isolating these parts from the whole will lead to the loss of the aesthetic representation. A translator as the special reader accumulates from discrete parts of information, which s/he synthesizes into a holistic whole, and then comes to analyze the parts against the gestalt whole. In this way, a translator may grasp the original systematically and aesthetically and appreciate the aesthetic charm of the original to acquire the similar aesthetic experience as possible as he could, based on which s/he can effectively represent the aesthetic qualities in the target text. Take an example from the translation of Li Shangyin's writings Ascending the Leyou Height (李商隐 《登乐游原》):

向晚意不适, 驱车登古原。夕阳无限好, 只是近黄昏。

xiangwan yi bushi, quche deng guyuan.

xiyang wuxian hao, zhishi jin huanghun.

"At dusk my heart is filled with gloom,

I drive my cab to ancient Tomb.

The setting sun appears sublime.

But oh! 'tis near its dying time."

(Translated by Xu Yuanchong)

The original version by Li Shangyin is thoroughly refined and aesthetically constructed: The aesthetic quality of harmony motivated by Li is delineated in logical frames by the coherent process of producing image- $\mathrm{G}$ as the bemoaning of declined Tang Dynasty, which is in a successively scenic progression rather than isolated image matching. The logical frames thus regulate at least the overall harmonious aesthetic quality and thereby affect the artistic quintessence of the splendid sunset at dusk that expresses the sense of alienation. That is, the aesthetically harmonious quality of image-G, which performs as a micro-framed unit and hence as an aesthetic reference point, is treated as complete or fundamental and as the crucial or definitional framing of the holistic image-G construction in English translation version.

$\mathrm{Xu}$ Yuanchong maintains a satellite-framed artistic quintessence to develop the TT for preserving the general aesthetically harmonious image-G syntactically while semantically evading the expression of unnecessarily culture-specific patterns. "heart is filled with gloom" is comparable to the original in the mood of the author filled with all unutterable and melancholy morose; "ancient Tomb" is working in concert with glooming to keep aesthetically unanimous with the emotional atmosphere of desolation and depression; "setting sun appears sublime" traces the correctness of sunsets splendid view of ST within a new imaginative blending of "dying time", which sets the author's mood of inwardly disappointments and frustrations. Xu adds luster to the equivalence of image-G's harmonious aesthetic quality by the use of the interjection "oh", which takes up the plaintive lament of the author in the TT.

\section{Aesthetic Representation of Image-G in English Translation of Chinese Poems}

\subsection{The Aesthetic Interaction between Image-G Schema and Cognitive Processing}

Image-G in Chinese poems actually processes certain aesthetic schema, which is not just from aesthetic qualities in imagery system, nor solely from a similar schema (both cognitive and aesthetic) underlying translators and readers' conceptual faculty, but rather from recurring patterns of homogeneous integration between them (Lefevere, 2004). Only when translators and authors' encyclopedic view and schematic representation is organically integrated in image- $G$ schema can aesthetic presentation of image-G be actualized in a successful way. When a translator's image-G schema is similar to that of the author, $s /$ he is in agreement with the aesthetic code and perceptual pattern of the author and thus his cognitive processing of image-G schema is to adjust in the ST to that of the TT presentation; when they do not have common image-G schema, the tacit comprehension cannot be achieved. The ST is the literary production of authors' mental actualization and his own image schema, while translators' cognitive processing of reproduction is stimulated by English and Chinese languages underlying two different schematic ideological backgrounds. In either of the cases, the aesthetic interaction 
between image-G schema and cognitive processing in general is to build on the holistic construction of image-G representation from S-text schemata to TT schemata.

\subsection{Translators' Conceptual Faculty That Is Conducive to Cognitive Processing}

Considering the interaction between textual environment and cognitive processing it is not surprising that there is a series of conceptual faculty which translators have possessed to function subjectively, and that a vital part of their psychological engagement system is in accord with such functioning. In attempting to formulate aesthetic representation of image- $G$ in translation process, translators' mental schema and conceptual faculty are inordinately critical in operating their cognition perception. According to Nida A. Eugene, imagination is a part of the mind's information processing system (Nida, 2001). Translators comprehend aesthetic significance of image- $G$ and represent aesthetic qualities not only on image- $G$ formulated mentally, but also based on the postulated conceptual faculty as following: association thinking is a conceptual faculty which is conducted by translators to merge together artistic qualities of image- $G$ who are not correspondingly associated and correlated between ST and TT. Varied artistic qualities are profoundly embedded in the two language schemata. As such, association thinking is necessary in experiencing the artistic qualities and formulating a gestalt image. Association thinking can help translators to interact with image-G qualities and reproduce image schemata and its metaphorical projections, and then coordinate conceptual image into an integrated and TT oriented whole.

\section{Conclusion}

The article makes an analysis of aesthetic actualization of gestalt image in English translation of Chinese poems based on gestalt psychology and cognitive linguistics. With a brief review of the exploration of image-G in Chinese poems, the study first investigates the aesthetic perception of image-G both as an inbred faculty and as an instruction for cognitive linguistic actualization. The study then elucidates the essentials of aesthetic actualization of image- $G$ in English translation of Chinese poems, namely, cognitive diagram dimension, aesthetic essence, aesthetic equivalence and aesthetic quality of harmony. It finally explores the aesthetic interaction and translators' conceptual faculty from the perspective of image-G representation. It could be concluded that the aesthetic actualization of gestalt image in English translation of Chinese poems is not just an abstract semantic process, but also should be understood as a conceptual mentalistic experience, which is more elementary than both concrete categories and abstract principle.

\section{References}

Barnstone, W. (1993). The poetics of Translation: History, Theory, Practice. New Haven: Yale University Press.

Bell, R. T. (1991). Translation and Translating: Theory and Practice. London: Longman Group UK Limited.

Croft, W., \& Cruse, A. D. (2004). Cognitive Linguistics. Cambridge: Cambridge University Press. https://doi.org/10.1017/CBO9780511803864

Dufrenne, P. M. (2002). The Phenomenology of Aesthetic Experience. Evanston: Northwestern University Press.

Fillmore, C. C. (1996). Frames and the Semantics of Understanding. Quaderni di Semantica, 6(2), 211-253.

Gibbs, R. W. (1995). The cognitive psychological reality of image schmas and their transformations, Cognitive Linguistics, 6, 96-273. https://doi.org/10.1515/cogl.1995.6.4.347

Jiang, Q. X. (2008). Aesthetic Progression in Literary Translation. Mata, 4, 860-871. https://doi.org/10.7202/019651ar

Jiang, Q. X. (2002). Aesthetic Progression in Literary Translation: Image-G Actualization. Beijing: The Commercial Press.

Koffka, K. (2015). Principle of Gestalt Psychology. Beijing: Peking University Press.

Kuang, F. T., \& Wen, X. (2003). Realization of Figure-Ground. Journal of Foreign Languages, 4, 24-31.

Langacker, R. W. (1987). Foundations of Cognitive Grammar (vol. 1): Theoretical Prerequisites. Stanford, CA: Stanford University Press.

Langacker, R. W. (1998). Conceptualization, symbolization and grammar. In M. Tomasello (Ed.), The New Psychology of labguage: Cognitive and functional Approaches to Language Structure (pp. 1-42). Mahwah, NJ: Laurence Erlaaum.

Langacker, R. W. (2004). Fountaions of cognitive grammar: Descriptive application (vol. 1). Beijing: Beijing University Press.

Lefevere, A. (2004). Translation, Rewriting, and the Manipulation of Literary Frame. Shanghai: Shanghai 
Foreign Language Education Press.

Liu, Z. D. (1992). Ten Lectures on Literary Translation. Beijing: China Translation \& Publishing Corporation.

Nida, E. A. (2001). Language, Culture and Translating. Shanghai: Shanghai Foreign Language Education Press.

Snell-Hornby, M. (1995). Translation Studies: An Integrated Approach (rev. ed.) Amsterdam/Philadelphia: John Benjamins.

Tabakowska, E. (1993). Cognitive Linguistics and Poetics of Translation. Tubingen: Guter Narr Verlag.

Wang, P. (2013). Imagery Study in Literary Translation. Chengdu: Southwestern University of Finance and Economics Press.

\section{Copyrights}

Copyright for this article is retained by the author(s), with first publication rights granted to the journal.

This is an open-access article distributed under the terms and conditions of the Creative Commons Attribution license (http://creativecommons.org/licenses/by/4.0/). 